\title{
A FORMAL SOLUTION OF CERTAIN DUAL INTEGRAL EQUATIONS
}

\author{
BY \\ CHARLES FOX
}

1. Introduction. Dual integral equations arise in many problems of mathematical physics, especially those with mixed boundary conditions. A well-known example is

$$
\begin{array}{ll}
\int_{0}^{\infty} u^{\alpha} J_{\mu}(u x) f(u) d u=g(x), & 0<x<1, \\
\int_{0}^{\infty} u^{\beta} J_{v}(u x) f(u) d u=h(x), & x>1,
\end{array}
$$

where $J_{\lambda}(x)$ is the usual Bessel function, $g(x)$ and $h(x)$ are given and $f(x)$ is to be found.

The case when $\alpha=\mu=v=0, \beta=1, g(x)$ is equal to a constant and $h(x)=0$ occurs in the problem of finding the electrostatic field arising from a flat circular disk charged to a constant potential. This was solved by Weber in 1873 and since then much complicated analysis [1], [3], [7], [8], has been developed to deal with the more general case (1), (2).

In this paper I deal with dual integral equations of a much more general nature than (1), (2). Instead of Bessel functions, the kernels will be $H$ functions of order $n$, defined below. These $H$ functions contain Bessel functions as special cases and my aim is to show that, with the help of a suitable terminology, it is possible to write down a solution by inspection. This solution has then to be verified by substitution, an operation which may prove to be quite difficult.

The $H$ functions of order $n$ used here are described in equations (3) and (4) below. On the left of (3) the $H$ function of order $n$ is expressed in abbreviated form, on the right of (3) it is expressed with all the constants displayed and on the right of (4) is given its definition:

$$
\begin{aligned}
H\left(x \mid \begin{array}{l}
\alpha_{i}, a_{i} \\
\beta_{i}, a_{i}
\end{array}: n\right) & =H\left(x \mid \begin{array}{l}
\alpha_{1}, a_{1} \\
\beta_{1}, a_{1}: \alpha_{2}, a_{2} \\
\beta_{2}, a_{2}
\end{array} \ldots \begin{array}{c}
\alpha_{n}, a_{n} \\
\beta_{n}, a_{n}
\end{array}\right) \\
& =\frac{1}{2 \pi i} \int_{c} \prod_{i=1}^{n}\left\{\frac{\Gamma\left(\alpha_{i}+s a_{i}\right)}{\Gamma\left(\beta_{i}-s a_{i}\right)}\right\} x^{-s} d s .
\end{aligned}
$$

Received by the editors October 2, 1964. 
We assume that the following conditions are satisfied; some of these conditions are necessary and some serve to simplify the problem.

(i) $a_{i}, \alpha_{i}, \beta_{i}$ are all real, $i=1,2, \cdots, n$.

(ii) $a_{i}>0, i=1,2, \cdots, n$.

(iii) Let $s=\sigma+i t$, where $\sigma$ and $t$ are real; then the contour $C$ along which the integral of (4) is taken is the straight line whose equation is $\sigma=\sigma_{0}$, where $\sigma_{0}$ is a constant. This line is parallel to the imaginary axis in the complex $s$ plane.

(iv) All the poles of the integrand of (4) are simple and lie to the left of the line $\sigma=\sigma_{0}$. This requires $\sigma_{0}>-\alpha_{i} / a_{i}, i=1,2, \cdots, n$.

Together with these conditions we require either (va) or (vb) below:

(va) $2 \sigma_{0} \sum_{i=1}^{n} a_{i}<\sum_{i=1}^{n}\left(\beta_{i}-\alpha_{i}\right)$,

(vb) $2 \sigma_{0} \sum_{i=1}^{n} a_{i}<\sum_{i=1}^{n}\left(\beta_{i}-\alpha_{i}\right)-1$.

The integral of (4), taken along the line $\sigma=\sigma_{0}$, converges if (va) holds and converges absolutely if (vb) holds. This can be verified by using the asymptotic expansion of the Gamma function.

The asymptotic expansion also shows that the contour $C$ of (4) can be closed by a large semicircle on the left. On computing the residues we then find that the $H$ function of order $n$ can be expressed as the sum of $n$ power series, the $i$ th of which is multiplied by $x^{\alpha_{i} / a_{i}}$. Each of these power series is an entire function.

In particular, for the $H$ function of order 1 we have

$$
H\left(\left.x\right|_{\beta, \frac{1}{2}} ^{\alpha, \frac{1}{2}}: 1\right)=2 x^{\alpha-\beta+1} J_{\alpha+\beta-1}(2 x),
$$

where $J$ denotes a Bessel function.

The dual integral equations we consider here are as follows:

$$
\int_{0}^{\infty} H\left(u x \mid \begin{array}{l}
\alpha_{i}, a_{i} \\
\beta_{i}, a_{i}
\end{array}: n\right) f(u) d u=g(x), \quad 0<x<1,
$$

and

$$
\int_{0}^{\infty} H\left(u x \mid \begin{array}{l}
\lambda_{i}, a_{i} \\
\mu_{i}, a_{i}
\end{array}: n\right) f(u) d u=h(x), \quad x>1
$$

where $g(x)$ and $h(x)$ are given and $f(x)$ is to be determined. Note that the constants $a_{i}, i=1,2, \cdots, n$, are the same for both (6) and (7).

We assume that the $H$ function of (6) satisfies the five conditions above and that the $H$ function of (7) satisfies these conditions with $\alpha_{i}$ replaced by $\lambda_{i}$ and $\beta_{i}$ replaced by $\mu_{i}, i=1,2, \cdots, n$. We also assume that a common value of $\sigma_{0}$ can be found for both the $H$ functions. When $n=1$ the dual equations (6) and (7) reduce to (1) and (2) as a very special case, by using (5).

Our aim is to find a formal solution of (6), (7) which can be written down by inspection of these equations. The solution, which makes use of the terminology of fractional integration, is given by equation (36). 
2. The Mellin transform. We make much use of the Mellin transform. We denote the Mellin transform of $f(u)$ by $\mathfrak{M}[f(u)]$ and if $\mathfrak{M}[f(u)]=F(s)$ we shall write $f(u)=\mathfrak{M}^{-1}[F(s)]$.

Formally we have

$$
\mathfrak{M}[f(u)]=F(s)=\int_{0}^{\infty} f(u) u^{s-1} d u
$$

and

$$
\mathfrak{M}^{-1}[F(s)]=f(u)=\frac{1}{2 \pi i} \int_{C} F(s) x^{-s} d s,
$$

where the contour $C$, in (9), is usually a straight line parallel to the imaginary axis in the complex $s(=\sigma+i t)$ plane, with equation $\sigma=\sigma_{0}$.

Associated with these transforms is the Parseval theorem as follows: if $\mathfrak{M}[p(u)]=P(s)$ and $\mathfrak{M}[f(u)]=F(s)$ then

$$
\int_{0}^{\infty} p(u) f(u) d u=\frac{1}{2 \pi i} \int_{C} P(s) F(1-s) d s .
$$

Again the contour $C$ is some straight line whose equation is of the form $\sigma=\sigma_{0}$.

From (8) or (9) it is easy to deduce that if $f(u x)$ is considered to be a function of $u$ with $x$ as a parameter, where $x>0$, then

$$
\mathfrak{M}[f(u x)]=x^{-s} \mathfrak{M}[f(u)] .
$$

From (11) and (10) we may deduce that

$$
\int_{0}^{\infty} p(u x) f(u) d u=\frac{1}{2 \pi i} \int_{c} P(s) x^{-s} F(1-s) d s,
$$

and this is the form in which we shall use the Parseval theorem here.

Conditions for the validity of (8), (9) and (10) can be found in $[9, \S \S 1.29,3.17$ and 4.14].

From (4) and (9) we may infer that

$$
\mathfrak{M}\left[H\left(u \mid \begin{array}{c}
\alpha_{i}, a_{i} \\
\beta_{i}, a_{i}
\end{array}: n\right)\right]=\prod_{i=1}^{n}\left\{\frac{\Gamma\left(\alpha_{i}+s a_{i}\right)}{\Gamma\left(\beta_{i}-s a_{i}\right)}\right\}
$$

and this follows from (4) if condition (vb) of $\$ 1$ holds [9, Theorem 29, p. 46]. It may still be true, however, if only condition (va) holds.

On using $\mathfrak{M}[f(u)]=F(s)$ we may apply the Parseval theorem (12) to (6) and (7) and, from (13), rewrite these equations in the forms

$$
\frac{1}{2 \pi i} \int_{c} \prod_{i=1}^{n}\left\{\frac{\Gamma\left(\alpha_{i}+s a_{i}\right)}{\Gamma\left(\beta_{i}-s a_{i}\right)}\right\} x^{-s} F(1-s) d s=g(x), \quad 0<x<1,
$$




$$
\frac{1}{2 \pi i} \int_{c} \prod_{i=1}^{n}\left\{\frac{\Gamma\left(\lambda_{i}+s a_{i}\right)}{\Gamma\left(\mu_{i}-s a_{i}\right)}\right\} x^{-s} F(1-s) d s=h(x), \quad x>1 .
$$

We assume that the contour $C$ is the same straight line $\sigma=\sigma_{0}$ for both (14) and (15).

(14) and (15) can be deduced from (6) and (7) only if we know something about the properties of $f(x)$ and since these are not known at present we must proceed formally. The method used here works more easily with (14) and (15) than with (6) and (7).

3. The reduction of (15) and (16) to equations with a common kernel. In dealing with dual integral equations it is customary to transform the given equations into two others with a common kernel and the problem is then reduced to solving one integral equation. Our procedure here is to transform the denominator of (14) to that of (15) and the numerator of (15) to that of (14).

In order to transform $\Gamma\left(\beta_{n}-s a_{n}\right)$ of (14) to $\Gamma\left(\mu_{n}-s a_{n}\right)$ of (16) we make use of the Beta function. If $b_{n}=1 / a_{n}$

$$
\begin{aligned}
\int_{0}^{x}\left(x^{b_{n}}-v^{b_{n}}\right)^{\mu_{n}-\beta_{n}-1} v^{b_{n} \beta_{n}-1-s} d v & \\
= & \frac{\Gamma\left(\mu_{n}-\beta_{n}\right)}{b_{n}} x^{b_{n} \mu_{n}-b_{n}-s} \frac{\Gamma\left(\beta_{n}-s a_{n}\right)}{\Gamma\left(\mu_{n}-s a_{n}\right)} .
\end{aligned}
$$

Certain conditions of convergence are required for (16), $\mu_{n}>\beta_{n}$ and $b_{n} \beta_{n}$ $=\beta_{n} / a_{n}>\sigma_{0}\left(s=\sigma_{0}+i t\right.$ on the line $\left.\sigma=\sigma_{0}\right)$. When fractional integration is introduced the first of these conditions may no longer be necessary and the second may be relaxed.

In (14) replace $x$ by $v$, multiply by $\left(x^{b_{n}}-v^{b_{n}}\right)^{\mu_{n}-\beta_{n}-1} v^{b_{n} \beta_{n}-1}$, where $b_{n}=1 / a_{n}$, and integrate through the integral sign with respect to $v$ from 0 to $x$, where $0<x<1$. We obtain

$$
\begin{aligned}
\frac{1}{2 \pi i} \int_{c} \prod_{i=1}^{n-1}\left\{\frac{\Gamma\left(\alpha_{i}+s a_{i}\right)}{\Gamma\left(\beta_{i}-s a_{i}\right)}\right\} \frac{\Gamma\left(\alpha_{n}+s a_{n}\right)}{\Gamma\left(\mu_{n}-s a_{n}\right)} x^{-s} F(1-s) d s \\
=\frac{b_{n}}{\Gamma\left(\mu_{n}-\beta_{n}\right)} x^{-b_{n} \mu_{n}+b_{n}} \int_{0}^{x}\left(x^{b_{n}}-v^{b_{n}}\right)^{\mu_{n}-\beta_{n}-1} v^{b_{n} \beta_{n}-1} g(v) d v
\end{aligned}
$$

where $0<x<1$ and $b_{n}=1 / a_{n}$.

We now introduce the first operator of fractional integration, denoted by $\mathfrak{I}$. When operating on $w(x)$ the operation is written as on the left of (18) below and defined as on the right of (18):

$$
\mathfrak{I}[\gamma, \varepsilon: m: w(x)]=\frac{m}{\Gamma(\gamma)} x^{-\varepsilon-m \gamma+m-1} \int_{0}^{x}\left(x^{m}-v^{m}\right)^{\gamma-1} v^{\varepsilon} w(v) d v .
$$


The case $m=1$ has been studied in detail by Kober [5] and the formulae for the more general case when $m>0$ has been given by Erdelyi [2]. There is, however, no essential difference between these two cases as can be seen from the formula

$$
\mathfrak{I}[\gamma, \varepsilon: m: w(x)]=\mathfrak{I}\left[\gamma, m^{-1}(\varepsilon+1)-1: 1: W(X)\right],
$$

where $x^{m}=X, v^{m}=V$ and $w(x)=W(X)$.

The operator $\mathfrak{I}$ exists if $w(x) \in L_{p}(0, \infty), p \geqq 1, \gamma>0, \varepsilon>(1-p) / p[2, \S 2]$. If, in addition, $w(x)$ can be differentiated sufficiently often then the operator $\mathfrak{I}$ exists for negative as well as positive values of $\gamma[2, \S 2$, p. 222].

For brevity we write (note $a_{i}^{-1}=b_{i}$ )

$$
\mathfrak{I}\left[\left(\mu_{i}-\beta_{i}\right),\left(\beta_{i} a_{i}^{-1}-1\right): a_{i}^{-1}: w(x)\right]=\mathfrak{I}_{i}[w(x)]
$$

and then it is evident that the right-hand side of (17) is equal to $\mathfrak{I}_{n}[g(x)]$, with $0<x<1$. In other words we can transform $\Gamma\left(\beta_{i}-s a_{i}\right)$, in (14), to $\Gamma\left(\mu_{i}-s a_{i}\right)$ if, at the same time, we apply the operator $\mathfrak{I}_{i}$ to the right of (14). Consequently, on transforming step by step for $i=n,(n-1), \cdots, 2,1$, we obtain from (14)

$$
\begin{aligned}
\frac{1}{2 \pi i} \int_{c} \prod_{i=1}^{n}\left\{\frac{\Gamma\left(\alpha_{i}+s a_{i}\right)}{\Gamma\left(\mu_{i}-s a_{i}\right)}\right\} x^{-s} F(1-s) d s \\
=\mathfrak{I}_{1}\left[\mathfrak{I}_{2} \cdots \mathfrak{I}_{n}[g(x)] \cdots\right], \quad 0<x<1 .
\end{aligned}
$$

The $\mathfrak{I}$ operators of (21) are commutative.

We next transform the numerator of (15) to that of (14) and for this purpose make use of the Beta function (note $b_{n}=1 / a_{n}$ as before)

$$
\begin{aligned}
\int_{x}^{\infty}\left(v^{b_{n}}-x^{b_{n}}\right)^{\lambda_{n}-\alpha_{n}-1} v^{b_{n}-b_{n} \lambda_{n}-s-1} d v & \\
& =\frac{\Gamma\left(\lambda_{n}-\alpha_{n}\right)}{b_{n}} x^{-b_{n} \alpha_{n}-s} \frac{\Gamma\left(\alpha_{n}+s a_{n}\right)}{\Gamma\left(\lambda_{n}+s a_{n}\right)} .
\end{aligned}
$$

For convergence of (22) we require $\lambda_{n}>\alpha_{n}$ at the lower limit and $a_{n} \sigma_{0}+\alpha_{n}>0$ at the upper limit. But when the fractional integration operator $\Omega$ is introduced some of these conditions may no longer be necessary. Here, as before, $\sigma_{0}$ is the real part of $s$ and also $x>0$.

Now replace $x$ by $v$ in (15), multiply by $\left(v^{b_{n}}-x^{b_{n}}\right)^{\lambda_{n}-a_{n}-1} v^{b_{n}-b_{n} \lambda_{n}-1}$ and integrate from $x$ to $\infty$, with respect to $v$, through the integral sign. The result is

$$
\begin{aligned}
& \frac{1}{2 \pi i} \int_{C} \prod_{i=1}^{n-1}\left\{\frac{\Gamma\left(\lambda_{i}+s a_{i}\right)}{\Gamma\left(\mu_{i}-s a_{i}\right)}\right\} \frac{\Gamma\left(\alpha_{n}+s a_{n}\right)}{\Gamma\left(\mu_{n}-s a_{n}\right)} x^{-s} F(1-s) d s \\
& =\frac{b_{n}}{\Gamma\left(\lambda_{n}-\alpha_{n}\right)} x^{b_{n} \alpha_{n}} \int_{x}^{\infty}\left(v^{b_{n}}-x^{b_{n}}\right)^{\lambda_{n}-\alpha_{n}-1} v^{b_{n}-b_{n} \lambda_{n}-1} h(v) d v,
\end{aligned}
$$

together with $x>1$ and $b_{n}=1 / a_{n}$. 
We now introduce the second operation of fractional integration denoted by $\Omega$ which, when operating upon $w(x)$, is written as on the left of (24) below and defined by the expression on the right of (24):

$$
\Re[\gamma, \varepsilon: m: w(x)]=\frac{m}{\Gamma(\gamma)} x^{\varepsilon} \int_{x}^{\infty}\left(v^{m}-x^{m}\right)^{\gamma-1} v^{-\varepsilon-m \gamma+m-1} w(v) d v .
$$

As in the case of $\mathfrak{I}$, if $w(x) \in L_{p}(0, \infty), p \geqq 1$, and $w(x)$ can be differentiated sufficiently often then $\Re$ exists if $m>0, \varepsilon>-1 / p$ while $\gamma$ can take any positive or negative value. The references for the operator $\mathfrak{I}$, of (18), give detailed information concerning $\Re$. There are, in fact, simple relations between $\boldsymbol{R}$ acting on $w(x)$ and $\mathfrak{I}$ acting upon $w(1 / x)$, see also (39).

For brevity we write

$$
\mathfrak{R}\left[\left(\lambda_{i}-\alpha_{i}\right), \alpha_{i} a_{i}^{-1}: a_{i}^{-1}: w(x)\right]=\Omega_{i}[w(x)]
$$

and then it is evident that the right-hand side of (23) is $\Omega_{n}[h(x)]$, where $x>1$. Consequently we can transform the factor $\Gamma\left(\lambda_{i}+s a_{i}\right)$ in (15) to $\Gamma\left(\alpha_{i}+s a_{i}\right)$ if, at the same time, the operator $\boldsymbol{\Re}_{i}$ is applied to the right-hand side of (15). On transforming step by step for $i=n,(n-1), \cdots, 2,1$ we obtain from (15)

$$
\frac{1}{2 \pi i} \int_{C} \prod_{i=1}^{n}\left\{\frac{\Gamma\left(\alpha_{i}+s a_{i}\right)}{\Gamma\left(\mu_{i}-s a_{i}\right)}\right\} x^{-s} F(1-s) d s
$$

Finally on writing

$$
=\boldsymbol{R}_{1}\left[\boldsymbol{\Omega}_{2} \cdots \boldsymbol{R}_{n}[h(x)] \cdots\right], \quad x>1 .
$$

$$
k(x)=\begin{array}{ll}
\mathfrak{I}_{1}\left[\mathfrak{I}_{2} \cdots \mathfrak{I}_{n}[g(x)] \cdots\right], & 0<x<1, \\
\mathfrak{N}_{1}\left[\mathfrak{\Re}_{2} \cdots \mathfrak{\Re}_{n}[h(x)] \cdots\right], & x>1
\end{array}
$$

we have, from (21) and (26)

$$
\frac{1}{2 \pi i} \int_{C} \prod_{i=1}^{n}\left\{\frac{\Gamma\left(\alpha_{i}+s a_{i}\right)}{\Gamma\left(\mu_{i}-s a_{i}\right)}\right\} x^{-s} F(1-s) d s=k(x) .
$$

(28) is the reduction of (14) and (15) to two equations with a common kernel. We can use the Parseval theorem of (12) to replace the left-hand side by an integral involving the product of an $H$ function of order $n$ and $f(u)$, but it is easier to work with (28) as it stands.

4. The formal solution of (6) and (7). We must now solve (28) for $f(x)=\mathfrak{M}^{-1}[F(s)]$, using the terminology of $\S 2$. For this purpose we use the generalized Fourier transform which consists of the reciprocity

$$
\begin{aligned}
& \phi(x)=\int_{0}^{\infty} p(u x) f(u) d u, \\
& f(x)=\int_{0}^{\infty} q(u x) \phi(u) d u .
\end{aligned}
$$


The functions $p(x)$ and $q(x)$ are known as the kernels and the transformation is said to be symmetrical if $p(x)=q(x)$ and unsymmetrical otherwise. A full and detailed account of this transform is given in [9, Chapter 8, especially $§ 8.9$ ].

Not every pair of functions can form the kernels of a transform such as (29), (30). With the Mellin transform notation of $\S 2$ let $\mathfrak{M}[p(u)]=P(s)$ and $\mathfrak{M}[q(u)]$ $=Q(s)$. Then among the conditions required for the validity of (29), (30) is the satisfaction of the functional equation

$$
P(s) Q(1-s)=1 \text {. }
$$

In addition to this $P(s)$ and $Q(s)$ must be bounded and regular in certain strips of the complex $s$-plane parallel to the imaginary axis and also $f(x)$ or $\phi(x)$ must belong to $L_{p}(0, \infty)$ for some $p \geqq 1$. Since our method is formal we need consider (31) only here.

We apply the Parseval theorem, (12) of $\S 2$, to the right-hand sides of (29) and (30). Writing $\mathfrak{M}[f(u)]=F(s)$, from (29) we obtain

$$
\phi(x)=\frac{1}{2 \pi i} \int_{C} P(s) x^{-s} F(1-s) d s .
$$

Also, on writing $\mathfrak{M}[\phi(u)]=\Phi(s)$ and using (31) we deduce from (30) that

$$
f(x)=\frac{1}{2 \pi i} \int_{c} \frac{1}{P(1-s)} x^{-s} \Phi(1-s) d s .
$$

Hence, if $P(s)$ and $\phi(x)$ are known in (32) we can solve for $f(x)=\mathfrak{M}^{-1}[F(s)]$ by means of equation (33).

On applying this idea to $(28)$ and writing $\mathfrak{M}[k(x)]=K(s)$ we deduce that

$$
f(x)=\frac{1}{2 \pi i} \int_{C} \prod_{i=1}^{n}\left\{\frac{\Gamma\left(\mu_{i}-a_{i}+s a_{i}\right)}{\Gamma\left(\alpha_{i}+a_{i}-s a_{i}\right)}\right\} x^{-s} K(1-s) d s .
$$

This is the formal solution of (14) and (15) and many important properties of $f(x)$ can be obtained from it. But, by using the Parseval theorem, (12) of $\S 2$, we can transform the integral of (34) so that the equation takes the form

$$
f(x)=\int_{0}^{\infty} H\left(u x \mid \begin{array}{l}
\mu_{i}-a_{i}, a_{i} \\
\alpha_{i}+a_{i}, a_{i}
\end{array}: n\right) k(u) d u,
$$

where $k(x)$ is given by (27). From conditions (iv) and (va) of $\$ 1$ the $H$ function of (35) exists if we can find a constant $\sigma_{0}$ such that $\sigma_{0} a_{i}>a_{i}-\mu_{i}, i=1,2, \cdots, n$, and $2 \sigma_{0} \quad \sum_{i=1}^{n} a_{i}<\sum_{i=1}^{n}\left(2 a_{t}+\alpha_{i}-\mu_{i}\right)$.

Written out in full (35) becomes

$$
\begin{aligned}
& f(x)=\int_{0}^{1} H\left(u x \mid \begin{array}{l}
\mu_{i}-a_{i}, a_{i} \\
\alpha_{i}+a_{i}, a_{i}
\end{array}: n\right) \mathfrak{I}_{1}\left[\mathfrak{I}_{2} \cdots \mathfrak{I}_{n}[g(u)] \cdots\right] d u \\
& +\int_{1}^{\infty} H\left(u x \mid \begin{array}{l}
\mu_{i}-a_{i}, a_{i} \\
\alpha_{i}+a_{i}, a_{i}
\end{array}: n\right) \boldsymbol{\Omega}_{1}\left[\Re_{2} \cdots \boldsymbol{\Omega}_{n}[h(u)] \cdots\right] d u,
\end{aligned}
$$


where the $H$ function of order $n$ is defined in (3) and (4), $\mathfrak{I}_{i}$ is defined by (20) and (18) and $\mathfrak{K}_{i}$ by (25) and (24). It is evident that the formal solution given by (36) can be written down by inspection from (6) and (7).

In the case when $n=1$ we can compare our result with known solutions of (1), (2). A solution of (1), (2) for the case $\beta=0$ is given by Peters, [8, equations (3.1), (3.2), p. 7] and the solution is given by (3.8) [8, p. 10]. On writing $n=1$, $a_{1}=1 / 2, \alpha_{1}=(\mu+\omega) / 2, \beta_{1}=(\mu-\omega+2) / 2, \lambda_{1}=v / 2$ and $\mu_{1}=(v+2) / 2$ in (36) we obtain a formula which agrees completely with Peter's (3.8) of [8]. Our method is formal, however, and so does not give conditions for the validity of the solution.

5. Properties of the operators $\mathfrak{I}$ and $\mathfrak{R}$ and of the $H$ function. In this section $I$ consider, very briefly, some properties of $\mathfrak{I}$ and $\Omega$ and of the $H$ function of order $n$. These may be useful when verifying that, under suitable conditions, (35) or (36) is a solution of (6) and (7).

As in $\$ 2$ denote the Mellin transform by $\mathfrak{M}$. Then we have

$$
\mathfrak{M}[\mathfrak{I}[\gamma, \varepsilon: m: f(x)]]=\frac{\Gamma[(\varepsilon+1-s) / m]}{\Gamma[\gamma+(\varepsilon+1-s) / m]} \mathfrak{M}[f(x)]
$$

and

$$
\mathfrak{M}[\mathfrak{N}[\gamma, \varepsilon: m: f(x)]]=\frac{\Gamma[(\varepsilon+s) / m]}{\Gamma[\gamma+(\varepsilon+s) / m]} \mathfrak{M}[f(x)] .
$$

When $m=1$ proofs of (37) and (38) are given in [5, p. 203]. When $m>0$, we can deduce (37) from the case $m=1$ by means of (19). The equation for the $R$ operator analogous to (19) is

$$
\mathfrak{N}[\gamma, \varepsilon: m: w(x)]=\Re\left[\gamma, \varepsilon m^{-1}: 1: W(X)\right],
$$

where $x^{m}=X$ and $w(x)=W(X)$. When $m>0$ equation (38) can be deduced from the case $m=1$ by means of (39).

The methods used here are essentially based upon (37) and (38). We could have applied $\mathfrak{I}$ and $\mathfrak{R}$ directly to (6) and (7) respectively but it is quicker and better to apply them to (14) and (15) as we have done in $\S 3$. This is mainly because the various steps are more openly exposed and so are easier to follow.

The proofs of (37) and (38) described above require that $\gamma>0$ in both cases, but this restriction is easily removed. For, on applying the operator $\mathfrak{M}^{-1}$ to (37) we obtain an equation for $\mathfrak{I}$ in which the right-hand side frequently exists even when $\gamma$ is negative. We therefore define $\mathfrak{I}$, with negative $\gamma$, to be equal to the right-hand side of (37) after being operated on by $\mathfrak{M}^{-1}$, whenever this has a meaning. Similarly for $\mathfrak{K}$ with negative $\gamma$, obtained from (38). For $m=1$ the formulae for $\mathfrak{I}$ and $\mathfrak{K}$ are given in [3, (2.4), p. 688]. They are as follows.

With $\gamma$ negative, let $n$ be any positive integer such that $n+\gamma \geqq 0$; then 


$$
\mathfrak{\Im}[\gamma, \varepsilon: 1: f(x)]=x^{-\gamma-\varepsilon} \frac{d^{n}}{d x^{n}}\left\{x^{\gamma+\varepsilon+n} \mathfrak{I}[n+\gamma, \varepsilon: 1: f(x)]\right\}
$$

and

$$
\Re[\gamma, \varepsilon: 1: f(x)]=(-1)^{n} x^{\gamma+\varepsilon+n} \frac{d^{n}}{d x^{n}}\left\{x^{-\gamma-\varepsilon} \mathfrak{R}[n+\gamma, \varepsilon: 1: f(x)]\right\} .
$$

The general formulae for the case $m>0$ can be obtained from these results by using (19) and (39). In particular, when $\gamma=0$ we have, from (37) and (38)

$$
\mathfrak{I}[0, \varepsilon: m: f(x)]=f(x)
$$

and

$$
\Re[0, \varepsilon: m: f(x)]=f(x) .
$$

From (37) and (38) we can also find formulae for $\mathfrak{I}^{-1}$ and $\mathfrak{\Omega}^{-1}$. If we replace $\mathfrak{I}$ on the left of (37) by $\mathfrak{I}^{-1}$ we should expect the right-hand Gamma function ratio to become inverted. This gives us

$$
\mathfrak{I}^{-1}[\gamma, \varepsilon: m: f(x)]=\mathfrak{I}[-\gamma, \varepsilon+m \gamma: m: f(x)]
$$

and from (38)

$$
\mathfrak{R}^{-1}[\gamma, \varepsilon: m: f(x)]=\mathfrak{R}[-\gamma, \varepsilon+m \gamma: m: f(x)] .
$$

To complete (43) and (44) we make use of the formula for $\mathfrak{I}$ and $\mathfrak{R}$ with negative $\gamma$ derived from (40) and (41). For the case $m=1$ formulae for $\mathfrak{I}^{-1}$ and $\Omega^{-1}$ are given in $[3$, p. $688,(2.3)]$. The solution (35) can be verified by reversing all the steps from (6), (7) to (35) and establishing conditions which justify each of these reversals. For this procedure the formulae for $\mathfrak{I}^{-1}$ and $\mathfrak{A}^{-1}$ are needed in going back from (28) to (14) and (15).

In $\S 4$ the $H$ function of order $n$ has been used as a generalized Fourier kernel. For a discussion of the $H$ function as a symmetrical Fourier kernel see [4]. The symmetrical case occurs when $p(x)=q(x)$ in (29) and (30) and on comparing (28) and (34) we see that the symmetrical case occurs here when

$$
\mu_{i}=\alpha_{i}+a_{i}, \quad i=1,2, \cdots, n .
$$

When

$$
a_{1}=a_{2}=\cdots=a_{n}
$$

the $H$ function reduces to a Meijer $G$ function and the conditions under which two $G$ functions can form a pair of unsymmetrical Fourier kernels are given in [6]. When neither (45) nor (46) hold there appears to be no general investigations about $H$ functions as Fourier kernels. In this case the general results obtained in $[9, \S 8.9]$ may be useful for functions in $L_{2}$ space. 


\section{REFERENCES}

1. I. W. Busbridge, Dual integral equations, Proc. London Math. Soc. 44 (1938), 115-129.

2. A. Erdelyi, On some functional transformations, Universita e Politecnico di Torino, Rend. Sem. Mat. 10 (1950-51), 217-234.

3. A. Erdelyi and I. N. Sneddon, Fractional integration and dual integral equations, Canad. J. Math. 14 (1962), 685-693.

4. Charles Fox, The $G$ and $H$ functions as symmetrical Fourier kernels, Trans. Amer. Math. Soc. 98 (1961), 395-429.

5. H. Kober, On fractional integrals and derivatives, Quart. J. Math. 11 (1940), 193-211.

6. Roop Narain, The G-functions as unsymmetrical Fourier kernels, Proc. Amer. Math. Soc. 14 (1963), 271-277.

7. B. Noble, The solution of Bessel function dual integral equations by a multiplying factor method, Proc. Cambridge Philos. Soc. 59 (1963), 351-362.

8. A. S. Peters, Certain dual integral equations and Sonine's integrals, IMM-NYU 285. Institute of Mathematical Sciences, New York University, New York, 1961.

9. E. C. Titchmarsh, Theory of Fourier integrals, Oxford Univ. Press, Oxford, 1937.

MCGILl UNIVERSITY,

Montreal, Canada. 